\title{
In-situ Raman and Nuclear Magnetic Resonance Study of Trapped Lithium in the Solid Electrolyte Interface of Reduced Graphene Oxide
}

Wei Tang, ${ }^{1,2,4+}$ Bee-Min Goh ${ }^{1,2+}$, Mary Y. Hu, ${ }^{3}$ Chuan Wan, ${ }^{3}$ Bingbing Tian, ${ }^{1,2}$ Xuchu Deng, ${ }^{3}$ Chengxin Peng, ${ }^{1,2}$ Ming Lin, ${ }^{5}$ Jian Zhi Hu ${ }^{3, *}$, Kian Ping Loh ${ }^{1,2}$ *

${ }^{1}$ Department of Chemistry, National University of Singapore, 3 Science Drive 3, Singapore 117543

${ }^{2}$ Centre for Advanced 2D Materials (CA2DM) and Graphene Research Centre, National University of Singapore, 6 Science Drive 2, Singapore 117546

${ }^{3}$ Pacific Northwest National Laboratory, Richland, WA 99352, USA.

${ }^{4}$ NUS Graduate School for Integrative Sciences and Engineering, 28 Medical Drive \#05-01, Singapore 117597

${ }^{5}$ Institute of Materials Research and Engineering, 2 Fusionopolis Way, \#08-03

Innovis, Singapore 138634

Author emails:

W.T.: a0103404@u.nus.edu;

B.G.: bee-min.goh@newcastle.ac.uk;

M.H.: mary.hu@pnnl.gov; 
C.W.: chuan.wan@pnnl.gov;

B.T.: chmt@ nus.edu.sg;

X.D.: xuchu.deng@pnnl.gov;

C.P.: pchxxm@live.cn;

M.L.: m-lin@imre.a-star.edu.sg;

J.H.: Jianzhi.Hu@pnnl.gov;

K.L.: chmlohkp@nus.edu.sg.

\section{Corresponding Author}

* Kian Ping Loh, Email: chmlohkp@nus.edu.sg; Jian Zhi Hu, Email:

Jianzhi.Hu@pnnl.gov. 

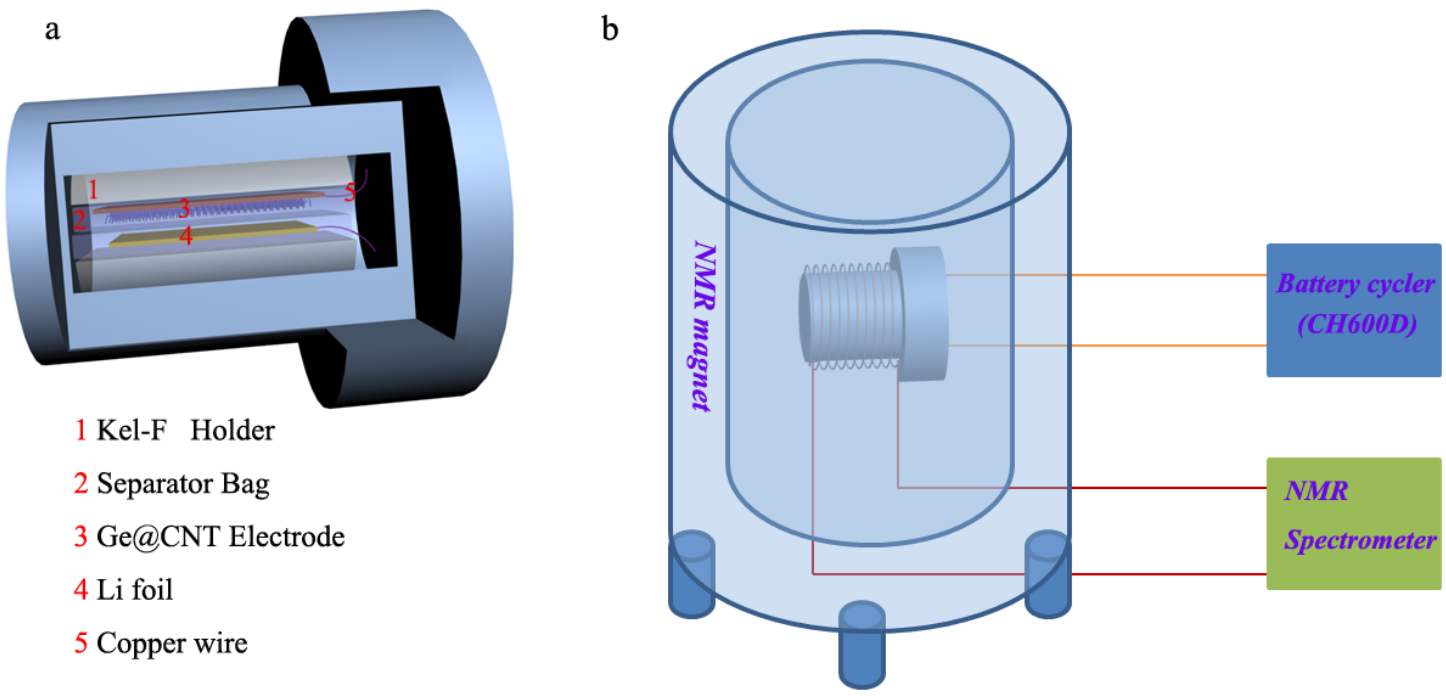

Figure S1. Illustration of the (a) in-situ NMR battery holder and (b) in-situ NMR experimental design. 
a
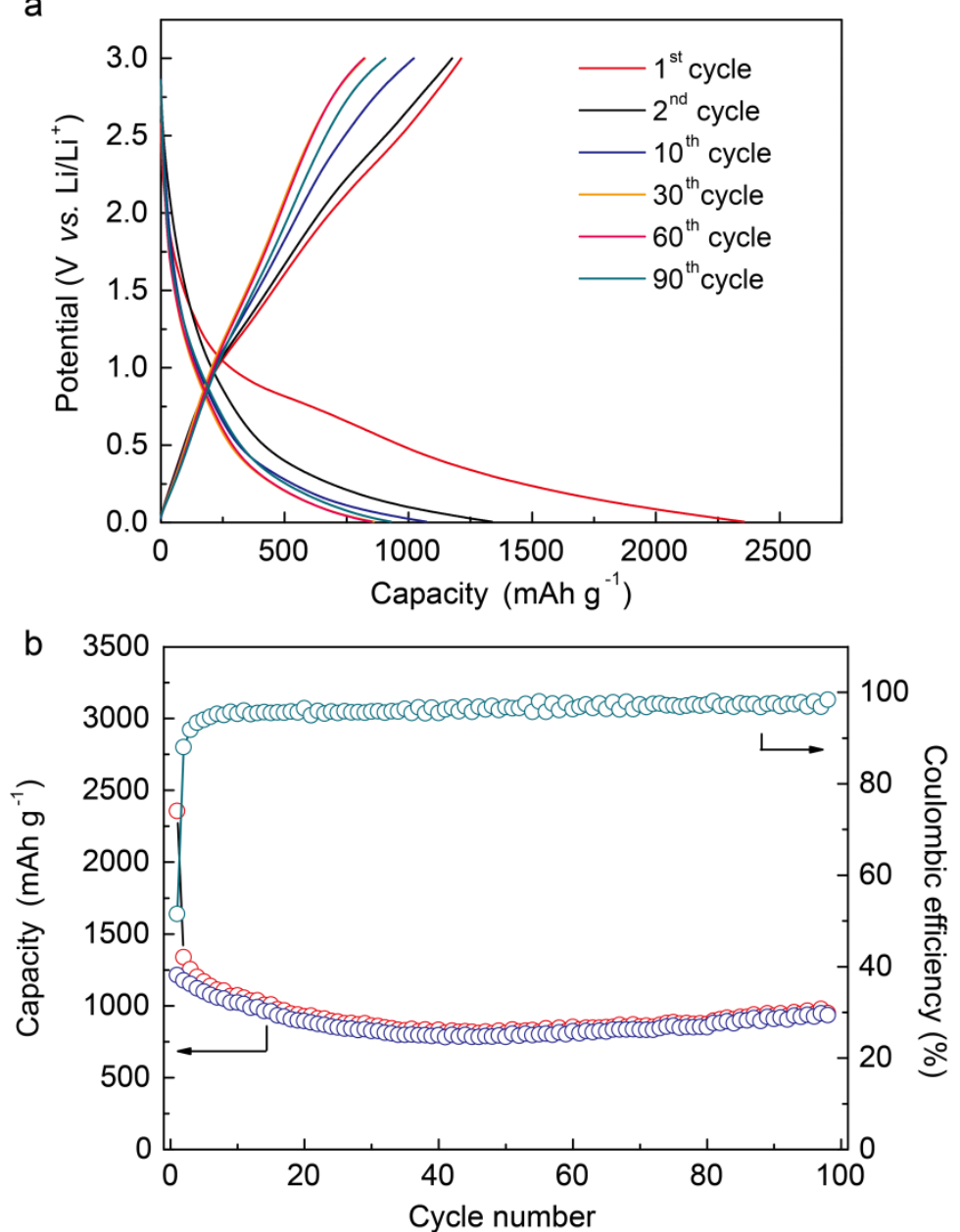

Figure S2 (a) Charge and discharge plot and (b) cyclability of rGO in the range of $0.005-3 \mathrm{~V}$ vs. $\mathrm{Li} / \mathrm{Li}^{+}$. 


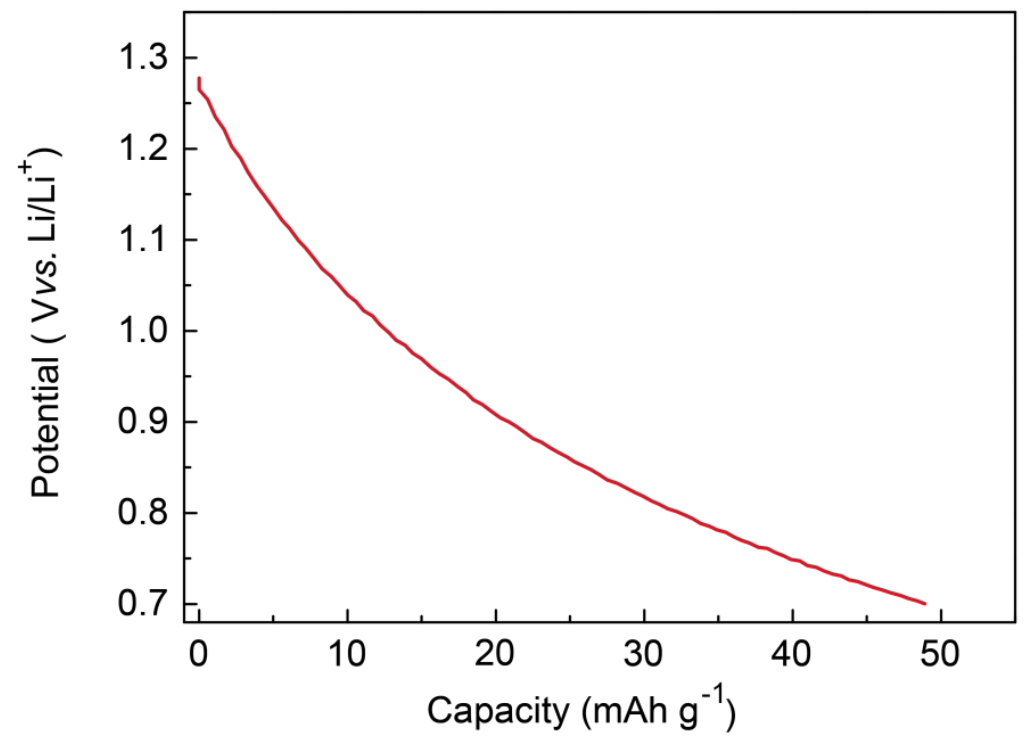

Figure S3 Re-discharge plot of rGO cell after remove electrical contact for 1600 minutes with the current density of $50 \mathrm{~mA} / \mathrm{g}$. 


\section{Prapartion of $\mathrm{rGO}$ :}

\section{Experimental details}

GO was prepared according to the modified method reported by Hummers (J. Am. Chem. Soc. 1958, 80, 1339). Reduced graphene oxide (RGO) was obtained by subjecting the as-prepared $\mathrm{GO}$ powder to annealing at $\mathrm{H} 2$ environment at $600{ }^{\circ} \mathrm{C}$ at ramp rate of $60^{\circ} \mathrm{C} / \mathrm{min}$ for 30 minutes.

Materials Characterizations: Scanning electron microscopy (SEM) analysis was performed on JEOL-6701F SEM. Raman spectra were collectted on Raman microscopy (Witech Alpha 300R, 532nm wavelength). FTIR of rGO and GO were performed in the reflection mode by using an OPUS/IR PS15 spectrometer (Bruker). The nitrogen adsorption-desorption isotherm experiment was conducted on Micromeritics ASAP 2020 V3.05 H. STM images were recorded in the constant current mode at RT after annealing the samples at specified temperature.

Electrochemical measurement: The electrochemical tests were performed using coin type 2016 half cell with lithium metal (Kyokuto Metal Co.,Ltd) as the counter and reference electrode and polypropylene film as a separator. The rGO electrodes were fabricated by mixing $70 \mathrm{wt} \%$ active materials, $10 \mathrm{wt} \%$ super p carbon black and $20 \mathrm{wt} \%$ PVDF binders in appropriate amount of NMP (N-methyl-2-pyrrolidinone, Sigma-Aldrich) as solvent. After stirring for 8 hours, the resulting paste was spread on a copper foil by Automatic Film Coater with Vacuum Pump \& Micrometer Doctor Blade (MTI). After evaporation of NMP solvent in a vacuum oven at $120{ }^{\circ} \mathrm{C}$ for $12 \mathrm{~h}$, the electrodes were pressed and cut into disks. The mass for each disk is around 1 $\mathrm{mg} / \mathrm{cm}^{2}$. Cells were assembled in argon-filled glove box (MBraun, Germany, $\mathrm{O}_{2}<$ 1ppm, $\mathrm{H}_{2} \mathrm{O}<1 \mathrm{ppm}$ ). Cyclic voltammetry studies were carried on an IVIUMnSTAT multichannel electrochemical ananlyser. The charge-discharge and cycling measurements were carried out using the Battery Test Stations (BitrodeCorp. St. Louis Mo USA. Model: MCV 16-0.5/0.01-5) at room temperature about $23^{\circ} \mathrm{C}$. Three-electrode cell (El-cell, Germany) was used for in-situ Raman study of rGO by using lithium metal as reference electrode.

NMR experiment: The working electrodes were coated on cupper foil with thickness of $50 \mu \mathrm{m}$. The area density of rGO composite is little larger than the electrodes using in the coin cell study (around $1.5 \mathrm{mg} / \mathrm{cm}^{2}$ ). Celgard 2500 separator (thickness of 25 $\mu \mathrm{m})$ was made into the shape of a bag to hold the working electrode inside to isolate the Li metal electrode (thickness of $750 \mu \mathrm{m}$ ) and the working electrode. The battery cell was inserted into a specially designed in-situ NMR battery capsule case made of Kel-F, also named as PCTFE (PolyChloroTriFluoroEthylene) that can hold a planar battery with a tight seal of the battery components to prevent the exposure of the battery to air. The details of this new in-situ battery holder will be published separately. The planar battery components were arranged such that the metal surface (both cupper and Li-metal foil) is perpendicular to the external main magnetic field, $\mathrm{B}_{0}$, and the direction of the excitation magnetic field, $\mathrm{B}_{1}$, is parallel to both the cupper and the Li-metal surface. A cupper wire with diameter of $0.1 \mathrm{~mm}$ was linked to the copper foil of the working electrode via three needle holes at one end of the copper foil to create a good and secure electrical connection. A second cupper wire of the 
same diameter was imbedded into the Li-metal electrode. Both wires were guided to the outside of the battery for charging and discharging of the in situ NMR battery via a CH600D instrument (Chronopotentiometry). The contents of the electrolytes were EC (Ethylene carbonate), DMC (dimethyl carbonate), DEC (diethyl carbonate) with volume ratios of $4: 2: 4$, and $1 \mathrm{M} \mathrm{LiFP}_{6}$. The in situ battery was assembled in an argon-filled glove box by pre-drying the electrode components at $60{ }^{\circ} \mathrm{C}$ under vacuum in glove box chamber for 12 hours before assembling.

\section{Full name list of reference 3 and 30:}

3. Hassoun, J.; Bonaccorso, F.; Agostini, M.; Angelucci, M.; Betti, M. G.; Cingolani, R.; Gemmi, M.; Mariani, C.; Panero, S.; Pellegrini, V.; Scrosati, B.

30. Hu, Y.-Y.; Liu, Z.; Nam, K.-W.; Olaf, J. B.; Cheng, J.; Xiao, H.; Matthew, T. D.; Xiqian, Y.; Kamila, M. W.; Lin-Shu, D.; Karena, W. C.; Peter, J. C.; Xiao-Qing, Y.; Clare, P. G. 